\section{A Call to Action to Track Generic Drug Quality Using Real-World Data and the FDA's Sentinel Initiative}

The invited perspectives in the May 2020 issue of JMCP on the "Safety and Quality of the Generic Drug Supply Chain" should be eye opening to this growing concern for the United States and the global drug supply. While the discussion across the 4 commentators focused on the history and clinical perspectives and outlined some steps moving forward, ${ }^{1-4}$ recent research in generic drug quality funded through ongoing FDA initiatives are worth mentioning, with additional context on the FDA's use of real-world data for their surveillance efforts.

As the number of manufacturing facilities has increased overall, especially internationally, the FDA has relied on a "Site Selection Model (SSM)" to identify high-risk facilities for inspection. The SSM includes site characteristics, compliance and inspection histories, product characteristics, and "hazard signals." Hazard signals are from a myriad of sources that may indicate potential product concerns and include FDA inspection reports, drug recalls, or signals from the FDA Adverse Event Reporting System (FAERS) and the MedWatch program. However, the FDA and manufacturers identify limitations of FAERS data to track generic drug quality, thus, innovations are desperately needed.

Towards this innovation, the FDA recognizes the potential for real-world data in the postmarketing surveillance period. Two recent studies funded by the Office of Generic Drugs exemplify potential approaches to surveil generic drug quality using administrative claims data. One study used the FDA's Sentinel Initiative distributed data network to track patient persistence to therapy, stratified by manufacturer, and revealed clear differences between manufacturers' products. ${ }^{5}$ The other study from my own group also tracked clinical events related to the indications and side effects of a specific drug (metoprolol succinate extended release) and compared the brand with 2 generic versions that were eventually recalled during the initial generic marketing period (2007-2008). ${ }^{6}$ In that study, we were able to capture stronger signals related to specific outcome events (e.g., heart attack and hypotension) compared with utilization metrics (i.e., persistence) and showed that these signals were strongest in the first 90 days of use.

Each of these studies represent opportunities for use of real-world data, specifically claims data, to strengthen generic drug quality research and surveillance with the possibility of near-real time evidence generation. The FDA Sentinel Initiative is the prime resource for postmarketing surveillance, and the onus is now on the relevant groups within the FDA, such as the Office of Generic Drugs and the Office of Pharmaceutical Quality, to use this resource to its fullest potential. In addition, managed care organizations with direct data access for their own members could also answer the call to use their own resources to ensure patient safety. These efforts and the resulting real-world evidence should be communicated to the FDA and be used as another tool to protect the U.S. drug supply and public health. Physicians, pharmacists, and other providers should likewise take patient concerns, or their own observations, seriously and add these experiences to the regulator's armament of data to combat the generic drug quality issue.

\section{Joshua D. Brown, PharmD, MS}

Center for Drug Evaluation and Safety,

Department of Pharmaceutical Outcomes and Policy,

College of Pharmacy, University of Florida, Gainesville

joshua.brown@ufl.edu

\section{DISCLOSURES}

The author reports funding from the U.S. Food and Drug Administration to study real-world data approaches to detect generic drug quality issues. There are no further conflicts or disclosures.

\section{REFERENCES}

1. Lever HM. A physician's perspective on generic drug quality. J Manag Care Spec Pharm. 2020;26(5):592-93. Available at: https://www.jmcp.org/ doi/10.18553/jmcp.2020.26.5.592

2. Hussar DA. Is the quality of generic drugs cause for concern? J Manag Care Spec Pharm. 2020;26(5):597-99. Available at: https://www.jmcp.org/ doi/10.18553/jmcp.2020.26.5.597.

3. Gaugh D. Global pharmaceutical manufacturing: association for accessible medicines perspective. J Manag Care Spec Pharm. 2020;26(5):594-96. Available at: https://www.jmcp.org/doi/10.18553/jmcp.2020.26.5.594.

4. Eban K. Can we trust the quality of generic drugs? J Manag Care Spec Pharm. 2020;26(5):589-91. Available at: https://www.jmcp.org/doi/10.18553/ jmcp.2020.26.5.589.

5. Gagne JJ, Popovic JR, Nguyen M, et al. Evaluation of switching patterns in FDA's Sentinel System: a new tool to assess generic drugs. Drug Saf. 2018;41(12):1313-23

6. Brown JD, Henriksen C, Vozmediano V, Schmidt S. Real-world data approaches for early detection of potential safety and effectiveness signals for generic substitution: a metoprolol extended-release case study. J Clin Pharmacol. 2019;59(9):1275-84.

Flash Continuous Glucose Monitoring in Primary Care Settings: The Need for Measurement of Novel Glycemic Metrics to Adopt Policy and Practice Change

The article "Effect of Pharmacist-Driven Professional Continuous Glucose Monitoring in Adults with Uncontrolled Diabetes" published in the May issue of JMCP by Sherrill et al. highlights how pharmacists can collaborate with physicians in primary care settings through use of flash continuous glucose monitoring (CGM). ${ }^{1}$ The authors sought to determine the most effective method for lowering hemoglobin Alc (HbAlc) at 6 months among 1 or 2 CGM interpretation encounters with a pharmacist as compared with 1 interpretation encounter with a physician. Clinical interventions were also described based on the encounters. The authors should be commended for undertaking this study, especially in light of only 1 other study published in the literature evaluating outcomes from pharmacist-driven CGM services. ${ }^{2}$ Additionally, the authors included patients with diabetes using oral antidiabetic medications, in 
whom flash CGM is not well studied but used frequently in clinical practice.

However, important considerations must be mentioned in interpreting the results and should be considered in the broad sense of the need for seeking policy and practice change to allow more patients with diabetes to take advantage of flash CGM. First, the authors used professional flash CGM, which is blinded to the patient. Although this was likely driven by payer considerations, unblinded CGM is more appropriate for the vast majority of patients. Unblinded flash CGM allows for glucose data to be viewable by the patient immediately and, when combined with diabetes education, can empower patients to recognize how diet, physical activity, and medications affect glucose levels. ${ }^{3}$

Second, the benefits to CGM monitoring include ability to assess additional glycemic metrics beyond $\mathrm{HbAlc}$, such as time in range, time below and above range, glycemic variability, and magnitude and frequency of glucose fluctuations. It appears that the authors made pharmacologic and nonpharmacologic interventions based on this CGM data but did not report these as outcomes. In order to enact changes to policy, which will increase coverage of flash CGM in a broader diabetes population by payers, these metrics must be reported as clinical research outcomes in studies evaluating flash CGM.

Finally, an important limitation in assessing the intervention is lack of comparison with a clinic without a pharmacist presence. Therefore, the results should be interpreted in light of the potential bias from how a pharmacist presence can influence clinical decision making among providers. It was also not mentioned if patients in the physician group had a pharmacist involved in their care during the study time frame.

While the value of a pharmacist-driven CGM service is not being questioned, careful consideration should be given to applying these findings in practice. Investigators are urged to follow best practices in CGM outcomes reporting, which have been previously reported. ${ }^{4}$ Prospectively, randomized studies evaluating outcomes with unblinded flash CGM in a team-based care setting involving a pharmacist are needed to demonstrate that CGM metrics can be used in clinical decision making and correlate with meaningful diabetes-related outcomes.

\section{Kevin Cowart, PharmD, MPH, BCACP, CDCES}

Department of Pharmacotherapeutics \& Clinical Research, Taneja College of Pharmacy, and Department of Internal Medicine, Morsani College of Medicine, University of South Florida kcowart2@usf.edu

\section{DISCLOSURES}

No outside funding supported the writing of this letter. The author has nothing to disclose.

\section{REFERENCES}

1. Sherrill CH, Houpt CT, Dixon EM, Richter SJ. Effect of pharmacist-driven professional continuous glucose monitoring in adults with uncontrolled diabetes. J Manag Care Spec Pharm. 2020;26(5):600-09. Available at: https:// www.jmcp.org/doi/full/10.18553/jmcp.2020.26.5.600.

2. Van Dril E, Schumacher C. Impact of professional continuous glucose monitoring by clinical pharmacists in an ambulatory care setting. J Am Coll Clin Pharm. 2019;2(6):638-44.

3. Hermanns N, Ehrmann D, Schipfer M, Kroger J, Haak T, Kulzer B The impact of a structured education and treatment programme (FLASH) for people with diabetes using a flash sensor-based glucose monitoring system: results of a randomized controlled trial. Diabetes Res Clin Pract. 2019;150:111-21.

4. Schnell O, Barnard K, Bergenstal R, et al. Role of continuous glucose monitoring in clinical trials: recommendations on reporting. Diabetes Technol Ther. 2017;19(7):391-99.

\section{The Authors Respond}

Thank you for the letter and interest regarding our article, "Effect of Pharmacist-Driven Professional Continuous Glucose Monitoring in Adults with Uncontrolled Diabetes," published in the May 2020 issue of JMCP. ${ }^{1}$ We appreciate the feedback and the opportunity to respond.

We agree that unblinded personal continuous glucose monitoring (CGM) is appropriate for many patients, is the future of home monitoring of glucose levels, and requires more research to promote policy and practice change to bring these services to all patients who could benefit from them. We do maintain, however, that there are many scenarios when blinded professional CGM can also be used. The main reason often cited is related to cost, as mentioned in the previous letter, since this approach can save the patient money while also providing a lucrative reimbursement potential for the clinic. ${ }^{2}$ Some clinicians may also use it as a first step to see if a CGM-naive patient may be interested in and willing to use CGM before making the jump to personal CGM. Additionally, professional CGM can be useful for patients with low health literacy or those simply not interested in the level of education and training needed to appropriately and safely use personal CGM. Ziegler et al. (2020) mention several other justifications for intermittent use of CGM that might be applied to professional CGM. ${ }^{3}$ Finally, we would argue that professional CGM can also be used to educate and empower patients regarding the effects of medication adherence, diet, and physical activity, although anecdotal, and can do so in a clinician-guided manner to promote safe changes and avoid dangerous glucose excursions resulting from patient-initiated changes. We do not contend the value of unblinded personal CGM, but highlight that of professional CGM.

Regarding the comment about CGM report metrics, since this study focused on CGM implementation as an intervention, rather than studying device accuracy, comparing with plasma glucose values, or exploring current glucose trends within a 